\title{
Resection of Cerebellopontine Angle Meningioma via Retrosigmoid Approach Aiming for Hearing Improvement
}

\author{
Yuki Shinya $^{1}$ Hideaki Ono $^{1}$ Tomohiro Inoue ${ }^{2} \quad$ Akira Tamura $^{1}$ \\ ${ }^{1}$ Department of Neurosurgery, Fuji Brain Institute and Hospital, \\ Shizuoka, Japan \\ 2 Department of Neurosurgery, NTT Medical Center Tokyo, Tokyo, Japan \\ Address for correspondence Hideaki Ono, MD, PhD, Department of \\ Neurosurgery, Fuji Brain Institute and Hospital, 270-12, Sugita, \\ Fujinomiya-City, Shizuoka, 418-0021, Japan \\ (e-mail: hideono-tky@umin.ac.jp).
}

J Neurol Surg B 2019;80(suppl S3):S302-S303.

\author{
Abstract \\ Keywords \\ - cerebellopotine angle \\ meningioma \\ - retrosigmoid \\ approach \\ - hearing improvement
}

We present a 71-year-old female case of left cerebellopontine angle (CPA) meningioma who presented with progressive hearing loss. The tumor was $35 \mathrm{~mm}$ in maximum diameter, obviously compressed the brain stem and cerebellum, and also displaced cranial nerves 7 th and 8 th anteriorly (-Fig. 1). Retrosigmoid approach was chosen to resect the tumor aiming for hearing improvement. We performed dissection of the tumor from cranial nerves 7 th and 8th gently and resection of the tumor except for the part adhesive to these cranial nerves (-Fig. 2). Postoperative course was good without any new neurological deficit. Postoperative examination also showed improvement of high-frequency hearing of the left side, and auditory brainstem response demonstrated wave 2 to 5 , which was not identify on preoperative examination. These procedures enabled safe and effective resection of the tumor and contributed to hearing improvement.

The link to the video can be found at: https://youtu.be/hkRSCxtV3bY.
Conflict of Interest

None.

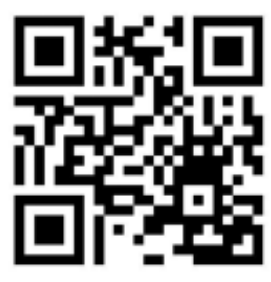

received

May 27, 2018

accepted after revision

November 11, 2018

published online

February 22, 2019

www.thieme.com/skullbasevideos

www.thieme.com/jnlsbvideos

DOI https://doi.org/

10.1055/s-0038-1676990.

ISSN 2193-6331. (c) 2019 Georg Thieme Verlag KG
Stuttgart · New York

License terms

c) $(1) \$$ 


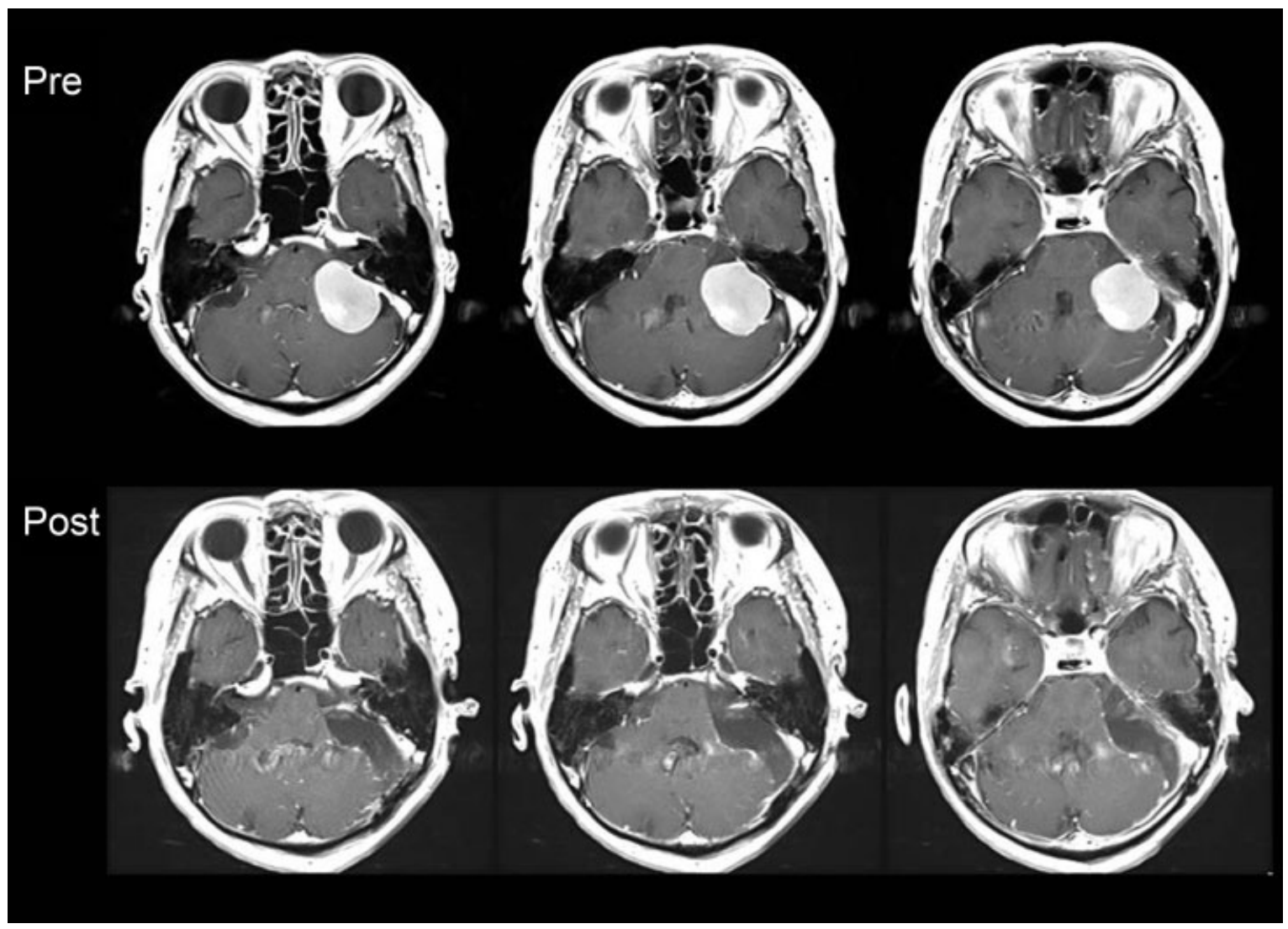

Fig. 1 Pre-and postoperative magnetic resonance imaging. Preoperative gadollinium enhanced magnetic resonance imaging (MRI) revealed meningioma at cerebellopontine angle, $35 \mathrm{~mm}$ in maximum diameter. Postoperative MRI showed gross total removal of the tumor, with slight enhancement along the cranial nerves 7th and 8th.

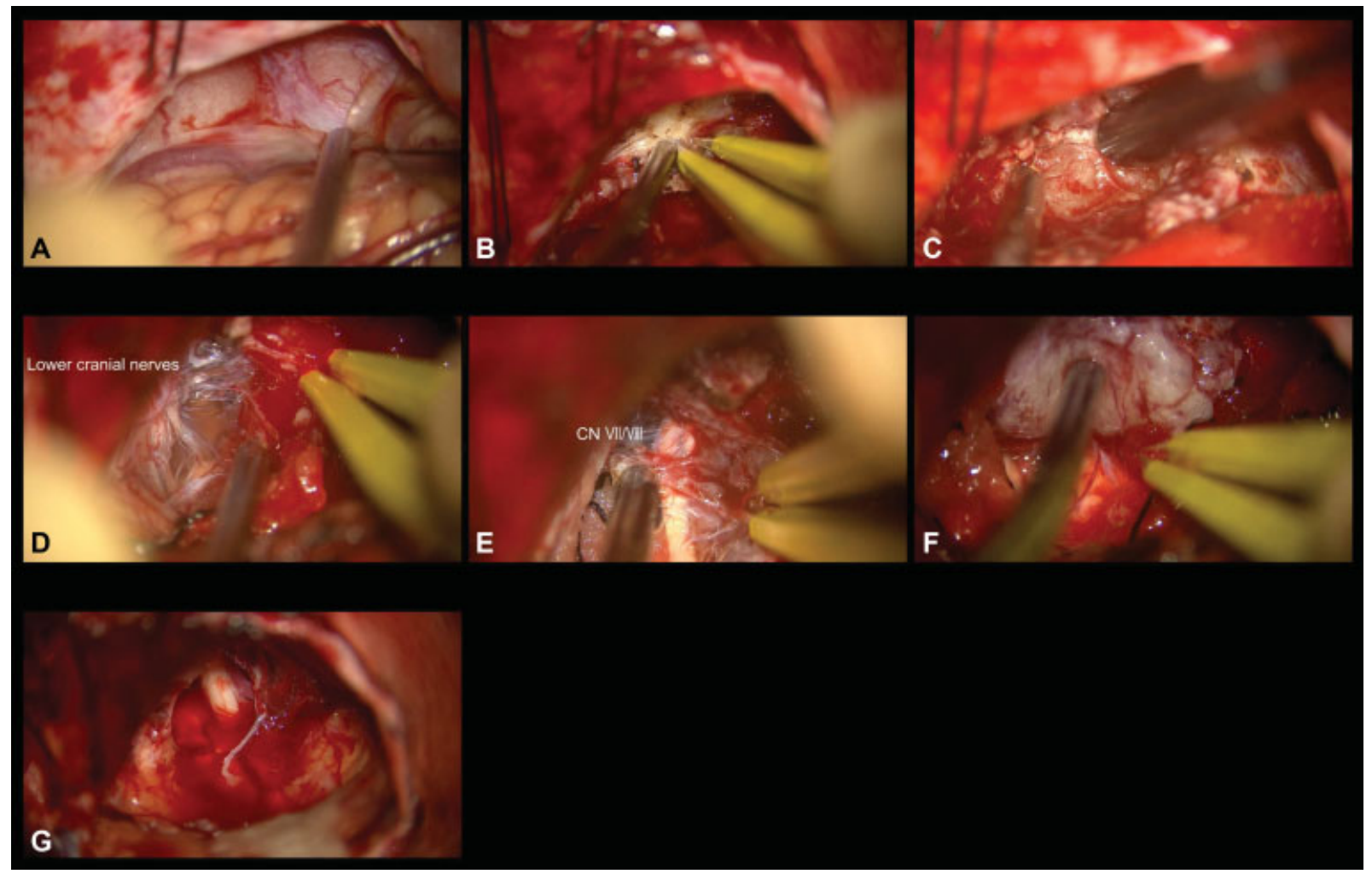

Fig. 2 Intraoperative images: (A) the arachnoid membrane was restored from the tumor; (B) detachment from petrous bone; (C) internal decompression; (D) dissection from lower cranial nerves; (E) detachment from cranial nerves 7th and 8th (F) detachment from brain stem and cerebellum; (G) gross total removal of the tumor, except for the adhesion to the cranial nerves 7 th and 8 th. $\mathrm{CN}$, cranial nerve. 